\title{
A Second-Order Eigen Theory for Static Electromagnetic Fields
}

\author{
Shaohua Guo \\ School of Civil Engineering and Architecture, Zhejiang University of Science and Technology, Hangzhou, China \\ Email: gsh606@yahoo.com.cn
}

Received March $28^{\text {th }}, 2010$; revised May $1^{\text {st }}, 2010$; accepted May $20^{\text {th }}, 2010$

\begin{abstract}
The static electromagnetic fields are studied here based on the standard spaces of the physical presentation, and the modal equations of static electromagnetic fields for anisotropic media are deduced. By introducing a set of new potential functions of order 2, several novel theoretical results were obtained: The classical potential functions of order 1 can be expressed by the new potential functions of order 2, the electric or magnetic potentials are scalar for isotropic media, and vector for anisotropic media. The amplitude and direction of the vector potentials are related to the anisotropic subspaces. Based on these results, we discuss the laws of static electromagnetic fields for anisotropic media.
\end{abstract}

Keywords: Anisotropic Media, Static Electromagnetic Field, Standard Spaces, Modal Equation Formatting

\section{Introduction}

By the Maxwell's electromagnetic field equations, we know that the electric and magnetic field are independent each other under the condition of static fields. The classical electromagnetic field theory also believes that the static electric field can be described by a scalar potential function, and the magnetic field by a vector one. Furthermore, for the passive region, the magnetic field can also be described by a scalar potential function $[1,2]$. But it should be pointed out that these results can only be obtained in the condition of isotropy, and are also only suit for the isotropic media. However, with the development of material science, more and more anisotropic dielectric or magnetic materials are applied to various fields, such as electron devices, communications and sensors, even for the traditional geological structure, we also can see the electrically anisotropic media or magnetically anisotropic media. It is found by recent research works that the limitations of classical static electromagnetic field theory have become obvious for these anisotropic media. For example, the above results for isotropic media don't exist for anisotropic media, even we don't know the definite form of the electric field potential function or magnetic field potential function, which make a great difficulty in solving the problem of anisotropic static electric or magnetic fields [3-5]. Unlike the classical static electromagnetic field theory, which studies the Maxwell's equations under the geometric representation, in this paper, the Maxwell's equations are restudied under the physical representation. As the result of this, the modal equations of static electric or magnetic fields are deduced, which give the novel expressions for the potential functions of static electric or magnetic fields for anisotropic media, and bring to light the intrinsic laws of static electromagnetic field.

\section{Standard Spaces of Electromagnetic Media}

In anisotropic electromagnetic media, the dielectric permittivity and magnetic permeability are tensors instead of scalars. The constitutive relations are expressed as follows

$$
\begin{aligned}
& D=\varepsilon \times E \\
& B=\mu \times H
\end{aligned}
$$

where the dielectric permittivity matrix $\varepsilon$ and the magnetic permeability matrix $\mu$ are usually symmetric ones, and the elements of the matrixes have a close relationship with the selection of reference coordinate. Suppose that if the reference coordinates is selected along principal axis of electrically or magnetically anisotropic media, the elements at non-diagonal of these matrixes turn to be zero. Therefore, Equations (1) and (2) are called the constitutive equations of electromagnetic media under the geometric presentation. Now we intend to get rid of effects of geometric coordinate on the constitutive 
equations, and establish a set of coordinate-independent constitutive equations of electromagnetic media under physical presentation. For this purpose, we solve the following problems of eigen-value of matrixes

$$
\begin{aligned}
& (\varepsilon-\lambda I) j=0 \\
& (\mu-\gamma I) \varphi=0
\end{aligned}
$$

where $\lambda_{i}(i=1,2,3)$ and $g_{i}(i=1,2,3)$ are respectively eigen dielectric permittivity and eigen magnetic permeability, which are constants of coordinate-independent. $j_{i}(i=1,2,3)$ and $\varphi_{i}(i=1,2,3)$ are respectively eigen electric vector and eigen magnetic vector, which show the electrically principal direction and magnetically principal direction of anisotropic media, and are all coordinate-dependent. We call these vectors as standard spaces. Thus, the matrix of dielectric permittivity and magnetic permeability can be spectrally decomposed as follows

$$
\begin{gathered}
\varepsilon=\Phi \Lambda \Phi^{\mathrm{T}} \\
\mu=\Psi \Pi \Psi^{\mathrm{T}}
\end{gathered}
$$

where $\Lambda=\operatorname{diag}\left[\lambda_{1}, \lambda_{2}, \lambda_{3}\right]$ and $\Pi=\operatorname{diag}\left[\gamma_{1}, \gamma_{2}, \gamma_{3}\right]$ are the matrix of eigen dielectric permittivity and eigen magnetic permeability, respectively. $\Phi=\left\{j_{1}, j_{2}, j_{3}\right\}$ and $\Psi=\left\{\varphi_{1}, \varphi_{2}, \varphi_{3}\right\}$ are respectively the modal matrix of electric media and magnetic media, which are both orthogonal and positive definite ones, and satisfy $\Phi^{T} \Phi=I, \quad \Psi^{T} \Psi=I$.

Projecting the electromagnetic physical qualities of the geometric presentation, such as the electric field intensity vector $E$, magnetic field intensity vector $H$, magnetic flux density vector $B$ and electric displacement vector $D$, into the standard spaces of the physical presentation, we get

$$
\begin{array}{rl}
D_{i}^{*}=j_{i}^{\mathrm{T}} \times D & i=1,2,3 \\
E_{i}^{*}=j_{i}^{\mathrm{T}} \times E & i=1,2,3 \\
B_{i}^{*}=\varphi_{i}^{\mathrm{T}} \times B & i=1,2,3 \\
H_{i}^{*}=\varphi_{i}^{\mathrm{T}} \cdot H & i=1,2,3
\end{array}
$$

These are the electromagnetic physical qualities under the physical presentation.

Substituting Equations (7)-(10) into Equations (1) and (2) respectively, and using Equations (5) and (6) yield, we have

$$
\begin{aligned}
& \left\{D^{*}\right\}=[\Lambda]\left\{E^{*}\right\} \\
& \left\{B^{*}\right\}=[\Pi]\left\{H^{*}\right\}
\end{aligned}
$$

or

$$
\begin{array}{ll}
D_{i}^{*}=\lambda_{i} E_{i}^{*} & i=1,2,3 \\
B_{i}^{*}=\gamma_{i} H_{i}^{*} & i=1,2,3
\end{array}
$$

The above equations are just the modal constitutive equations in the form of scalar.

\section{Matrix form of Static Electromagnetic Field Equation}

The classical static Maxwell's equations in passive region can be written as

$$
\begin{aligned}
& \nabla \times E=0, \nabla \cdot D=0 \\
& \nabla \times H=0, \nabla \cdot B=0
\end{aligned}
$$

where $\nabla$ is a Hamilton operator. It is seen from the above equations that the electric field and magnetic field are not only independent, but also the same in the form of equation. So, it is undistinguishable to study the problems of electric field or magnetic field under the static condition. For this purpose, we consider here only the problem of electric field.

From Equation (15), we can see that one is a vector equation, another is scalar one. It is well known that the vector equation can be written as the matrix one, but the scalar equation can not. By the first one of Equation (15), we have

$$
[\partial]\{E\}=0
$$

where

$$
[\partial]=\left[\begin{array}{ccc}
0 & -\partial_{z} & \partial_{y} \\
\partial_{z} & 0 & -\partial_{x} \\
-\partial_{y} & \partial_{x} & 0
\end{array}\right]
$$

It is an operator matrix of order 1 .

In order get the matrix expression of static electromagnetic equations, both of Equation (15) should be reformed in a suitable form.

For dynamic electromagnetic fields, a matrix equation of electromagnetic waves be dedued by author [6]

$$
[\Delta]\{E\}=-\nabla_{t}^{2}[\mu][\varepsilon]\{E\}
$$

where

$$
[\Delta]=[\partial][\partial]=\left[\begin{array}{ccc}
-\left(\partial_{z z}+\partial_{y y}\right) & \partial_{x y} & \partial_{x z} \\
\partial_{y x} & -\left(\partial_{x x}+\partial_{z z}\right) & \partial_{y z} \\
\partial_{z x} & \partial_{z y} & -\left(\partial_{x x}+\partial_{y y}\right)
\end{array}\right]
$$

It is an operator matrix of order 2. For static electromag-netic fields, we have

$$
[\Delta]\{E\}=0
$$

Now, rewriting the second one of Equation (15) in the 
index form of tensor

$$
D_{i^{\prime} i}=0
$$

Differentiating the above equation with index $j$, it become a vectorial one

$$
D_{i^{\prime} i j}=0
$$

Rewritting it in the matrix form, we get

$$
[\nabla]\{D\}=0
$$

where

$$
[\nabla]=\left[\begin{array}{lll}
\partial_{11} & \partial_{21} & \partial_{31} \\
\partial_{12} & \partial_{22} & \partial_{32} \\
\partial_{13} & \partial_{23} & \partial_{33}
\end{array}\right]
$$

In this paper, $[\Delta]$ and $[\nabla]$ is defined as the matrix of electic intensity and electic displacement operators respectively

\section{Eigen Equations of Static Electric Fields}

Now, we transform the matrix equations of static electric field into modal ones.

Substituting Equation (7) into Equation (21), and multiplying it with the transpose of modal matrix in left, we have

$$
[\Phi]^{T}[\Delta][\Phi]\left\{E^{*}\right\}=0
$$

It be proved [6] that the matrix of electric intensity operator can also be spectrally decomposed, that is

$$
[\Phi]^{T}[\Delta][\Phi]=\left[\Delta^{*}\right]
$$

Thus, Equation (26) can be uncoulped and become

$$
\left[\Delta^{*}\right]\left\{E^{*}\right\}=0
$$

or

$$
\Delta_{i}^{*} E_{i}^{*}=0 \quad i=1,2,3
$$

in which

$$
\Delta_{i}^{*}=\left\{\partial_{i}^{*}\right\}^{T} \cdot\left\{\partial_{i}^{*}\right\} \quad i=1,2,3
$$

where

$$
\left\{\partial_{i}^{*}\right\}=\left\{\phi_{i}\right\}^{T}[\partial]\left\{\phi_{i}\right\}
$$

In same way, substituting Equation (7) into Equation (24), and multiplying it with the transpose of modal matrix in left, we have

$$
[\Phi]^{T}[\nabla][\Phi]\left\{D^{*}\right\}=0
$$

let

$$
[\Phi]^{T}[\nabla][\Phi]=\left[\nabla^{*}\right]
$$

and substituting Equation (11) into Equation (32),we have

$$
\left[\nabla^{*}\right][\Lambda]\left\{E^{*}\right\}=0
$$

Comparing Equation.(34) with Eq.(28), we get

$$
\left[\nabla^{*}\right][\Lambda]=\left[\Delta^{*}\right]
$$

It is seen that $\left[\nabla^{*}\right]$ is also a diagonal matrix. We call it as eigen matrix of electric displacement operator. Thus, we have

$$
\nabla_{i}^{*} D_{i}^{*}=0 \quad i=1,2,3
$$

So, Equations (29) and (36) constitute of the eigen equations of static electric field. Different from the classical ones, they show the simplicity and symmetry of static electromagnetic law.

\section{General Solution of Eigen Equations of Static Electric Fields}

Let

$$
\begin{array}{rr}
E_{i}^{*}=\nabla_{i}^{*} \chi_{i} & i=1,2,3 \\
D_{i}^{*}=\Delta_{i}^{*} \chi_{i} & i=1,2,3
\end{array}
$$

where $\{\chi\}$ is an unknown row vector, which is new electric potential function of order 2 .

Substituting Equations (37) and (38) into Equations (29) and (36) respectively, a unified equation are obtained as follows

$$
\square_{i}^{*} \chi_{i}^{*}=0 \quad i=1,2,3
$$

where, $\square_{i}^{*}=\Delta_{i}^{*} \cdot \nabla_{i}^{*}(i=1,2,3)$ is $i$ th modal operator of electric field, and a differential operator of order 4. Deferent from the Laplce's equation for the classical electric potential function of order 1 , the new electric potential function of order 2 can be solved by the modal differential equation of higher order, and the classical electric potential function of order 1 can be expressed by the new electric potential function of order 2 . Once the modal potential functions are solved from Equation (41), the electric intensity and electric displacement can be obtained by the following conversion

$$
\begin{aligned}
& \{\boldsymbol{E}\}=\left\{\phi_{1}\right\} \nabla_{1}^{*} \chi_{1}+\left\{\phi_{2}\right\} \nabla_{2}^{*} \chi_{2}+\left\{\phi_{3}\right\} \nabla_{3}^{*} \chi_{3} \\
& \{\boldsymbol{D}\}=\left\{\phi_{1}\right\} \lambda_{1} \nabla_{1}^{*} \chi_{1}+\left\{\phi_{2}\right\} \lambda_{2} \nabla_{2}^{*} \chi_{2}+\left\{\phi_{3}\right\} \lambda_{3} \nabla_{3}^{*} \chi_{3}
\end{aligned}
$$

In order to get the classical electric potential function of order 1, we rewritting Equation (40) by using Equations (30) and (35)

$$
\{\boldsymbol{E}\}=\sum_{i}\left\{\phi_{i}\right\}\left\{\partial_{i}^{*}\right\}^{T}\left\{\partial_{i}^{*}\right\} \chi_{i}=\sum_{i}\left\{\partial_{i}^{*}\right\}\left(\left\{\phi_{i}\right\}\left\{\partial_{i}^{*}\right\}^{T} \chi_{i}\right)
$$


let

$$
\psi_{i}=-\left\{\phi_{i}\right\} \cdot\left\{\partial_{i}^{*}\right\}^{T} \chi_{i}
$$

It is just the electric potential function of order 1 for anisotropic media. Thus the electric intensity and electric displacement can be expressed by the electric potential function of order 1 as follows

$$
\begin{gathered}
\{\boldsymbol{E}\}=-\left\{\partial_{1}^{*}\right\} \psi_{1}-\left\{\partial_{2}^{*}\right\} \psi_{2}-\left\{\partial_{3}^{*}\right\} \psi_{3} \\
\{\boldsymbol{D}\}=-\left\{\partial_{1}^{*}\right\} \lambda_{1} \psi_{1}-\left\{\partial_{2}^{*}\right\} \lambda_{2} \psi_{2}-\left\{\partial_{3}^{*}\right\} \lambda_{3} \psi_{3}
\end{gathered}
$$

\section{The Modal Boundary Condition of Static Electric Field}

It is seen from above that in order to get the solutions of the electric intensity and electric displacement, we can turn to solving the modal potential functions. So, the modal Equation (39) should have the corresponding modal boundary condition.

An effective boundary case is: Electric displacement functions of two side of interface should be equal

$$
\left\{D^{(1)}\right\}=\left\{D^{(2)}\right\}
$$

or

$$
D_{i}^{(1)}=D_{i}^{(2)} \quad i=1,2,3
$$

Rewriting Equation (46) in the modal form, we have

$$
\left[\Delta^{(1)^{*}}\right]\left\{\chi^{(1)^{*}}\right\}=\left[\Delta^{(2)^{*}}\right]\left\{\chi^{(2)^{*}}\right\}
$$

or

$$
\Delta_{i}^{(1)^{*}} \chi_{i}^{(1)^{*}}=\Delta_{i}^{(2)^{*}} \chi_{i}^{(2)^{*}} \quad i=1,2,3
$$

and

$$
\lambda_{i}^{(1)} \nabla_{i}^{(1)^{*}} \chi_{i}^{(1)^{*}}=\lambda_{i}^{(2)} \nabla_{i}^{(2)^{*}} \chi_{i}^{(2)^{*}} \quad i=1,2,3
$$

\section{Application}

In this section, we discuss the laws of static electric field only in anisotropic dielectrics.

\subsection{Isotropic Crystal}

The matrix of dielectric permittivity of isotropic dielectrics is following

$$
\boldsymbol{\varepsilon}=\left[\begin{array}{lll}
\varepsilon_{11} & 0 & 0 \\
0 & \varepsilon_{11} & 0 \\
0 & 0 & \varepsilon_{11}
\end{array}\right]
$$

The eigen-values and eigen-vectors are respectively shown as below

$$
\Lambda=\operatorname{diag}\left[\varepsilon_{11}, \varepsilon_{11}, \varepsilon_{11}\right]
$$

$$
\boldsymbol{\Phi}=\left[\begin{array}{lll}
1 & 0 & 0 \\
0 & 1 & 0 \\
0 & 0 & 1
\end{array}\right]
$$

We can see from the above equations that there is only one eigen-space in isotropic crystal, which is a tripledegenerate one, and the space structure is following

$$
\boldsymbol{W}=W_{1}^{(3)}\left[\phi_{1}, \phi_{2}, \phi_{3}\right]
$$

The basic vector of one dimension in a triple-degenerate subspace is

$$
\phi^{*}=\frac{\sqrt{3}}{3}\{1,1,1\}^{T}
$$

The eigen electric displacement operator of isotropic crystal are

$$
\begin{aligned}
& \nabla_{1}^{*}=\frac{1}{3}\left(\partial_{1}^{2}+\partial_{2}^{2}+\partial_{3}^{2}\right) \\
& \left\{\partial_{1}^{*}\right\}=\frac{\sqrt{3}}{3}\left\{\partial_{1}, \partial_{2}, \partial_{3}\right\}
\end{aligned}
$$

Therefore, the static electric field equation in isotropic crystal can be written as below

$$
\left(\partial_{x}^{2}+\partial_{y}^{2}+\partial_{z}^{2}\right)^{2} \chi_{1}=0
$$

Thus, the electric strength and electric displacement of isotropic crystal become

$$
\{E\}=\left\{\phi_{1}\right\} \nabla_{1}^{*} \chi_{1}=\left(\partial_{1}^{2}+\partial_{2}^{2}+\partial_{3}^{2}\right)\left\{\begin{array}{l}
1 \\
1 \\
1
\end{array}\right\} \chi_{1}
$$

$$
\{\boldsymbol{D}\}=\left\{\phi_{1}\right\} \lambda_{1} \nabla_{1}^{*} \chi_{1}=\varepsilon_{11}\left(\partial_{1}^{2}+\partial_{2}^{2}+\partial_{3}^{2}\right)\left\{\begin{array}{l}
1 \\
1 \\
1
\end{array}\right\} \chi_{1}
$$

The classical electric potential function of order 1 is

$$
\psi_{1}=-\left(\partial_{1}+\partial_{2}+\partial_{3}\right) \chi_{i}
$$

So, the electric intensity and electric displacement of isotropic crystal can also be expressed by the classical electric potential function of order 1 as follows

$$
\{E\}=-\left\{\begin{array}{l}
\partial_{x} \\
\partial_{y} \\
\partial_{z}
\end{array}\right\} \psi_{1}
$$

or

$$
\boldsymbol{E}=-\boldsymbol{\nabla} \psi_{1}
$$




$$
\{D\}=-\varepsilon_{11}\left\{\begin{array}{l}
\partial_{x} \\
\partial_{y} \\
\partial_{z}
\end{array}\right\} \psi_{1}
$$

or

$$
\boldsymbol{D}=-\varepsilon_{11} \nabla \psi_{1}
$$

It is seen that Equations (62)-(65) are the same as the classical results, in which the electric potential is a scalar. But from the following analysis, we will see that only for isotropy we have same results as classical theory.

\subsection{Uniaxial Crystal}

The matrix of dielectric permittivity of uniaxial dielectrics is following

$$
\boldsymbol{\varepsilon}=\left[\begin{array}{lll}
\varepsilon_{11} & 0 & 0 \\
0 & \varepsilon_{11} & 0 \\
0 & 0 & \varepsilon_{33}
\end{array}\right]
$$

The eigen-values and eigen-vectors are respectively shown as below

$$
\begin{gathered}
\boldsymbol{\Lambda}=\operatorname{diag}\left[\varepsilon_{11}, \varepsilon_{11}, \varepsilon_{33}\right] \\
\boldsymbol{\Phi}=\left[\begin{array}{lll}
1 & 0 & 0 \\
0 & 1 & 0 \\
0 & 0 & 1
\end{array}\right]
\end{gathered}
$$

We can see from the above equations that there are two eigen-spaces in uniaxial crystal, one of which is a double-degenerate space, and the space structure is following

$$
\boldsymbol{W}=W_{1}^{(2)}\left[\phi_{1}, \phi_{2}\right] \oplus W_{2}^{1}\left[\phi_{3}\right]
$$

The basic vectors in two subspaces are following

$$
\begin{gathered}
\boldsymbol{\phi}_{1}^{*}=\frac{\sqrt{2}}{2}\{1,1,0\}^{T} \\
\boldsymbol{\phi}_{2}^{*}=\{0,0,1\}^{T}
\end{gathered}
$$

The eigen electric strength qualities of uniaxial crystal are respectively shown as below

$$
\begin{array}{r}
E_{2}^{*}=\boldsymbol{\phi}_{2}^{\mathrm{T}} \cdot \boldsymbol{E}=E_{3} \\
\boldsymbol{\phi}_{1}^{\mathrm{T}} E_{1}^{*}=\boldsymbol{E}-\boldsymbol{\phi}_{2}^{\mathrm{T}} E_{2}^{*}
\end{array}
$$

Multiplying Equation (57) with $\phi_{2}$, using $\phi_{2}^{\mathrm{T}} \cdot \boldsymbol{\phi}_{1}=0$ and $\boldsymbol{\phi}_{i}^{\mathrm{T}} \cdot \boldsymbol{\phi}_{i}=1(i=1,2)$, we get

$$
\left|E_{1}^{*}\right|=\sqrt{\left(\boldsymbol{E}-\boldsymbol{\phi}_{2}^{\mathrm{T}} E_{2}^{*}\right)^{\mathrm{T}}\left(\boldsymbol{E}-\boldsymbol{\phi}_{2}^{\mathrm{T}} E_{2}^{*}\right)}=\sqrt{E_{1}^{2}+E_{2}^{2}}
$$

The eigen electric displacement operators of uniaxial crystal are respectively shown as below

$$
\begin{gathered}
\nabla_{1}^{*}=\frac{1}{2}\left(\partial_{11}+\partial_{22}\right) \\
\nabla_{2}^{*}=\partial_{33} \\
\left\{\partial_{1}^{*}\right\}=\frac{\sqrt{2}}{2}\left\{\partial_{1}, \partial_{2}, 0\right\} \\
\left\{\partial_{2}^{*}\right\}=\left\{0,0, \partial_{3}\right\}
\end{gathered}
$$

Therefore, the static electric field equation in uniaxial crystal can be written as below

$$
\begin{gathered}
\left(\partial_{x}^{2}+\partial_{y}^{2}\right)^{2} \chi_{1}^{*}=0 \\
\partial_{z}^{4} \chi_{2}^{*}=0
\end{gathered}
$$

It is seen from Equations (79) and (80) that there are two static electric fields in uniaxial crystal. Thus, the electric intensity and electric displacement become

$$
\begin{aligned}
& \{E\}=\left\{\phi_{1}\right\} \nabla_{1}^{*} \chi_{1}+\left\{\phi_{2}\right\} \nabla_{2}^{*} \chi_{2} \\
& =\left\{\begin{array}{l}
1 \\
1 \\
0
\end{array}\right\}\left(\partial_{11}+\partial_{22}\right) \chi_{1}+\left\{\begin{array}{l}
0 \\
0 \\
1
\end{array}\right\} \partial_{33} \chi_{2} \\
& =\left\{\begin{array}{l}
\left(\partial_{11}+\partial_{22}\right) \chi_{1}^{*} \\
\left(\partial_{11}+\partial_{22}\right) \chi_{1}^{*} \\
\partial_{33} \chi_{2}^{*}
\end{array}\right\} \\
& \{D\}=\left\{\phi_{1}\right\} \lambda_{1} \nabla_{1}^{*} \chi_{1}+\left\{\phi_{2}\right\} \lambda_{2} \nabla_{2}^{*} \chi_{2} \\
& =\left\{\begin{array}{l}
\varepsilon_{11}\left(\partial_{11}+\partial_{22}\right) \chi_{1}^{*} \\
\varepsilon_{11}\left(\partial_{11}+\partial_{22}\right) \chi_{1}^{*} \\
\varepsilon_{33} \partial_{33} \chi_{2}^{*}
\end{array}\right\}
\end{aligned}
$$

The classical electric potential function of order 1 is

$$
\begin{gathered}
\psi_{1}=-\frac{1}{2}\left(\partial_{1}+\partial_{2}\right) \chi_{1} \\
\psi_{1}=-\partial_{3} \chi_{2}
\end{gathered}
$$

So, the electric intensity and electric displacement of uniaxial crystal can also be expressed by the classical electric potential function of order 1 as follows

$$
\begin{gathered}
\{E\}=-\left\{\begin{array}{l}
\partial_{1} \psi_{1} \\
\partial_{2} \psi_{1} \\
\partial_{3} \psi_{2}
\end{array}\right\} \\
\{D\}=-\left\{\begin{array}{l}
\varepsilon_{11} \partial_{x} \psi_{1} \\
\varepsilon_{11} \partial_{y} \psi_{1} \\
\varepsilon_{33} \partial_{z} \psi_{2}
\end{array}\right\}
\end{gathered}
$$

It is seen that the electric intensity and electric displacement of uniaxial crystal are quite different from 
those in isotropic crystal, and there exist two kinds of modal electric potential functions in uniaxial crystal, so they become vectorial ones, this is also different from the classical results of static electric field.

\subsection{Biaxial Crystal}

The matrix of dielectric permittivity of biaxial dielectrics is following

$$
\boldsymbol{\varepsilon}=\left[\begin{array}{lll}
\varepsilon_{11} & 0 & 0 \\
0 & \varepsilon_{22} & 0 \\
0 & 0 & \varepsilon_{33}
\end{array}\right]
$$

The eigen-values and eigen-vectors are respectively shown as below

$$
\begin{gathered}
\Lambda=\operatorname{diag}\left[\varepsilon_{11}, \varepsilon_{22}, \varepsilon_{33}\right] \\
\boldsymbol{\Phi}=\left[\begin{array}{lll}
1 & 0 & 0 \\
0 & 1 & 0 \\
0 & 0 & 1
\end{array}\right]
\end{gathered}
$$

We can see from the above equations that there are three eigen-spaces in biaxial crystal, and the space structure is following

$$
\boldsymbol{W}=W_{1}^{(1)}\left[\phi_{1}\right] \oplus W_{2}^{(1)}\left[\phi_{2}\right] \oplus W_{3}^{(1)}\left[\phi_{3}\right]
$$

The eigen-qualities and eigen electric displacement operators of biaxial crystal are respectively shown as below

$$
\begin{gathered}
E_{1}^{*}=\boldsymbol{\phi}_{1}^{\mathrm{T}} \cdot \boldsymbol{E}=E_{1} \\
E_{2}^{*}=\boldsymbol{\phi}_{2}^{\mathrm{T}} \cdot \boldsymbol{E}=E_{2} \\
E_{3}^{*}=\boldsymbol{\phi}_{3}^{\mathrm{T}} \cdot \boldsymbol{E}=E_{3} \\
\nabla_{1}^{*}=\partial_{11} \quad\left\{\partial_{1}^{*}\right\}=\left\{\partial_{1}, 0,0\right\}^{T} \\
\nabla_{2}^{*}=\partial_{22} \quad\left\{\partial_{2}^{*}\right\}=\left\{0, \partial_{2}, 0\right\}^{T} \\
\nabla_{3}^{*}=\partial_{33} \quad\left\{\partial_{3}^{*}\right\}=\left\{0,0, \partial_{3}\right\}^{T}
\end{gathered}
$$

Therefore, the static electric field equation in biaxial crystal can be written as below

$$
\begin{aligned}
& \partial_{x}^{4} \chi_{1}^{*}=0 \\
& \partial_{y}^{4} \chi_{2}^{*}=0 \\
& \partial_{z}^{4} \chi_{3}^{*}=0
\end{aligned}
$$

It is seen from Equations (97)-(99) that there are three static electric fields in biaxial crystal. Thus, the electric intensity and electric displacement become

$$
\begin{gathered}
\{E\}=\left\{\phi_{1}\right\} \nabla_{1}^{*} \chi_{1}+\left\{\phi_{2}\right\} \nabla_{2}^{*} \chi_{2}+\left\{\phi_{3}\right\} \nabla_{3}^{*} \chi_{3} \\
=\left\{\begin{array}{l}
1 \\
0 \\
0
\end{array}\right\} \nabla_{1}^{*} \chi_{1}+\left\{\begin{array}{l}
0 \\
1 \\
0
\end{array}\right\} \nabla_{2}^{*} \chi_{2}+\left\{\begin{array}{l}
0 \\
0 \\
1
\end{array}\right\} \nabla_{3}^{*} \chi_{3} \\
=\left\{\begin{array}{l}
\partial_{11} \chi_{1} \\
\partial_{22} \chi_{2} \\
\partial_{33} \chi_{3}
\end{array}\right\} \\
\{D\}=\left\{\begin{array}{l}
\varepsilon_{11} \partial_{11} \chi_{1} \\
\varepsilon_{22} \partial_{22} \chi_{2} \\
\varepsilon_{33} \partial_{33 z} \chi_{3}
\end{array}\right\}
\end{gathered}
$$

The classical electric potential function of order 1 is

$$
\begin{aligned}
& \psi_{1}=-\partial_{1} \chi_{1} \\
& \psi_{2}=-\partial_{2} \chi_{2} \\
& \psi_{3}=-\partial_{3} \chi_{2}
\end{aligned}
$$

So, the electric intensity and electric displacement of uniaxial crystal can also be expressed by the classical electric potential function of order 1 as follows

$$
\begin{gathered}
\{E\}=-\left\{\begin{array}{l}
\partial_{1} \psi_{1} \\
\partial_{2} \psi_{2} \\
\partial_{3} \psi_{3}
\end{array}\right\} \\
\{D\}=-\left\{\begin{array}{l}
\varepsilon_{11} \partial_{x} \psi_{1} \\
\varepsilon_{22} \partial_{y} \psi_{2} \\
\varepsilon_{33} \partial_{z} \psi_{3}
\end{array}\right\}
\end{gathered}
$$

It is seen that the electric intensity and electric displacement of biaxial crystal are quite different from those in isotropic crystal, and there exist three kinds of modal electric potential functions in uniaxial crystal, so they become vectorial ones, this is also different from the classical results of static electric field.

\subsection{Monoclinic Crystal}

The matrix of dielectric permittivity of monoclinic dielectrics is following

$$
\boldsymbol{\varepsilon}=\left[\begin{array}{lll}
\varepsilon_{11} & \varepsilon_{12} & 0 \\
\varepsilon_{12} & \varepsilon_{22} & 0 \\
0 & 0 & \varepsilon_{33}
\end{array}\right]
$$

The eigen-values and eigen-vectors are respectively shown as below

$$
\Lambda=\operatorname{diag}\left[\lambda_{1}, \lambda_{2}, \varepsilon_{33}\right]
$$




$$
\left\{\begin{array}{l}
\boldsymbol{\phi}_{1}=\frac{\varepsilon_{12}}{\sqrt{\left(\lambda_{1}-\varepsilon_{11}\right)^{2}+\varepsilon_{12}^{2}}}\left[\frac{\lambda_{1}-\varepsilon_{11}}{\varepsilon_{12}}, 1,0\right]^{\mathrm{T}} \\
\boldsymbol{\phi}_{2}=\frac{\varepsilon_{12}}{\sqrt{\left(\lambda_{2}-\varepsilon_{11}\right)^{2}+\varepsilon_{12}^{2}}}\left[1, \frac{\lambda_{2}-\varepsilon_{11}}{\varepsilon_{12}}, 0\right]^{\mathrm{T}} \\
\boldsymbol{\phi}_{3}=[0,0,1]^{\mathrm{T}}
\end{array}\right.
$$

where

$$
\lambda_{1,2}=\frac{\left(\varepsilon_{11}+\varepsilon_{22}\right)}{2} \pm \sqrt{\left[\frac{1}{2}\left(\varepsilon_{11}-\varepsilon_{22}\right)\right]^{2}+\varepsilon_{12}^{2}} \quad \lambda_{3}=\varepsilon_{33}
$$

We can see from the above equations that there are also three eigen-spaces in monoclinic crystal, and the space structure is following

$$
\boldsymbol{W}=W_{1}^{(1)}\left[\phi_{1}\right] \oplus W_{2}^{(1)}\left[\phi_{2}\right] \oplus W_{3}^{(1)}\left[\phi_{3}\right]
$$

The eigen-qualities and eigen electric displacement operators of monoclinic crystal are respectively shown as below

$$
\begin{gathered}
E_{1}^{*}=\boldsymbol{\phi}_{1}^{\mathrm{T}} \cdot \boldsymbol{E}=\frac{1}{\sqrt{\left(\lambda_{1}-\varepsilon_{11}\right)^{2}+\varepsilon_{12}^{2}}}\left[\left(\lambda_{1}-\varepsilon_{11}\right) E_{1}+\varepsilon_{12} E_{2}\right] \\
E_{2}^{*}=\boldsymbol{\phi}_{2}^{\mathrm{T}} \cdot \boldsymbol{E}=\frac{1}{\sqrt{\left(\lambda_{2}-\varepsilon_{11}\right)^{2}+\varepsilon_{12}^{2}}}\left[\varepsilon_{12} E_{1}+\left(\lambda_{2}-\varepsilon_{11}\right) E_{2}\right] \\
\left\{\begin{array}{l}
\nabla_{1}^{*}=a_{1}^{2} \partial_{11}+b_{1}^{2} \partial_{22}+2 a_{1} b_{1} \partial_{1} \partial_{2} \\
\left\{\partial_{1}^{*}\right\}=\left\{a_{1} \partial_{1}+b_{1} \partial_{2}, a_{1} \partial_{1}+b_{1} \partial_{2}, 0\right\}^{T} \\
\left\{\begin{array}{l}
\nabla_{2}^{*}=a_{2}^{2} \partial_{11}+b_{2}^{2} \partial_{22}+2 a_{2} b_{2} \partial_{1} \partial_{2} \\
\left\{\partial_{2}^{*}\right\}=\left\{a_{2} \partial_{1}+b_{2} \partial_{2}, a_{2} \partial_{1}+b_{2} \partial_{2}, 0\right\}^{T}
\end{array}\right. \\
\nabla_{3}^{*}=\partial_{33} \quad\left\{\partial_{3}^{*}\right\}=\left\{0,0, \partial_{3}\right\}^{T}
\end{array}\right.
\end{gathered}
$$

where

$$
b_{i}=\frac{\varepsilon_{12}}{\sqrt{\left(\lambda_{i}-\varepsilon_{11}\right)^{2}+\varepsilon_{12}^{2}}} \quad a_{i}=b_{i} \frac{\lambda_{i}-\varepsilon_{11}}{\varepsilon_{12}} \quad i=1,2
$$

Therefore, the static electric field equation in monoclinic crystal can be written as below

$$
\left(a_{1}^{2} \partial_{11}+b_{1}^{2} \partial_{22}+2 a_{1} b_{1} \partial_{1} \partial_{2}\right)^{2} \chi_{1}^{*}=0
$$

$$
\begin{gathered}
\left(a_{2}^{2} \partial_{11}+b_{2}^{2} \partial_{22}+2 a_{2} b_{2} \partial_{1} \partial_{2}\right)^{2} \chi_{2}^{*}=0 \\
\partial_{z}^{4} \chi_{3}^{*}=0
\end{gathered}
$$

It is seen from Equations (118)-(120) that there exist also three static electric fields in monoclinic crystal, which is a little different from the results in biaxial crystal because of the distortion of static electric fields. Thus, the electric intensity and electric displacement become

$$
\begin{aligned}
& \left\{\begin{array}{l}
\left.\left\{\begin{array}{l} 
\\
\left.a_{1} \partial_{1}+b_{1} \partial_{2}\right\} \\
a_{1} \partial_{1}+b_{1} \partial_{2} \\
0
\end{array}\right\}\left(a_{1}^{2} \partial_{11}+b_{1}^{2} \partial_{22}+2 a_{1} b_{1} \partial_{1} \partial_{2}\right) \chi_{1}\right\} \nabla_{2}^{*} \chi_{2}+\left\{\phi_{3}\right\} \nabla_{3}^{*} \chi_{3}= \\
+\left\{\begin{array}{l}
a_{2} \partial_{1}+b_{2} \partial_{2} \\
a_{2} \partial_{1}+b_{2} \partial_{2} \\
0
\end{array}\right\}\left(a_{2}^{2} \partial_{11}+b_{2}^{2} \partial_{22}+2 a_{2} b_{2} \partial_{1} \partial_{2}\right) \chi_{2} \\
+\left\{\begin{array}{l}
0 \\
0 \\
1
\end{array}\right\} \partial_{33} \chi_{3} \\
\left\{\begin{array}{l}
D\}=\left\{\phi_{1}\right\} \lambda_{1} \nabla_{1}^{*} \chi_{1}+\left\{\phi_{2}\right\} \lambda_{2} \nabla_{2}^{*} \chi_{2}+\left\{\phi_{3}\right\} \lambda_{3} \nabla_{3}^{*} \chi_{3}= \\
+\left\{\begin{array}{l}
a_{1} \partial_{1}+b_{1} \partial_{2} \\
a_{1} \partial_{1}+b_{1} \partial_{2} \\
0 \\
0
\end{array}\right\} \lambda_{1}\left(a_{1}^{2} \partial_{11}+b_{1}^{2} \partial_{22}+2 a_{1} b_{1} \partial_{1} \partial_{2}\right) \chi_{1} \chi_{3}
\end{array}\right\} \\
+\left\{\begin{array}{l}
a_{2} \partial_{1}+b_{2} \partial_{2} \\
a_{2} \partial_{1}+b_{2} \partial_{2} \\
0
\end{array}\right\} \lambda_{2}\left(a_{2}^{2} \partial_{11}+b_{2}^{2} \partial_{22}+2 a_{2} b_{2} \partial_{1} \partial_{2}\right) \chi_{2}
\end{array}\right. \\
& \left\{\begin{array}{l}
0 \\
1
\end{array}\right\}
\end{aligned}
$$

The classical electric potential function of order 1 is

$$
\begin{gathered}
\psi_{1}=-\left[a_{1}\left(a_{1} \partial_{1}+b_{1} \partial_{2}\right)+b_{1}\left(a_{1} \partial_{1}+b_{1} \partial_{2}\right)\right] \chi_{1} \\
\psi_{2}=-\left[a_{2}\left(a_{2} \partial_{1}+b_{2} \partial_{2}\right)+b_{2}\left(a_{2} \partial_{1}+b_{2} \partial_{2}\right)\right] \chi_{2} \\
\psi_{3}=-\partial_{3} \chi_{3}
\end{gathered}
$$

So, the electric intensity and electric displacement of monoclinic crystal can also be expressed by the classical electric potential function of order 1 as follows

$$
\{E\}=-\left\{\begin{array}{l}
a_{1} \partial_{1}+b_{1} \partial_{2} \\
a_{1} \partial_{1}+b_{1} \partial_{2} \\
0
\end{array}\right\} \psi_{1}-\left\{\begin{array}{l}
a_{2} \partial_{1}+b_{2} \partial_{2} \\
a_{2} \partial_{1}+b_{2} \partial_{2} \\
0
\end{array}\right\} \psi_{2}-\left\{\begin{array}{l}
0 \\
0 \\
\partial_{3}
\end{array}\right\} \psi_{3}
$$




$$
\begin{aligned}
& \{D\}=-\lambda_{1}\left\{\begin{array}{l}
a_{1} \partial_{1}+b_{1} \partial_{2} \\
a_{1} \partial_{1}+b_{1} \partial_{2} \\
0
\end{array}\right\} \psi_{1} \\
& -\lambda_{2}\left\{\begin{array}{l}
a_{2} \partial_{1}+b_{2} \partial_{2} \\
a_{2} \partial_{1}+b_{2} \partial_{2} \\
0
\end{array}\right\} \psi_{2}-\varepsilon_{33}\left\{\begin{array}{l}
0 \\
0 \\
\partial_{3}
\end{array}\right\} \psi_{3}
\end{aligned}
$$

It is seen that the electric intensity and electric displacement of biaxial crystal are aiso quite different from those in isotropic crystal.

\section{Conclusions}

In this paper, we construct the standard spaces under the physical presentation by solving the eigen-value problem of the matrixes of dielectric permittivity and magnetic permeability, in which we get the eigen dielectric permittivity and eigen magnetic permeability, and the corresponding eigen vectors. The former are coordinateindependent and the latter are coordinate-dependent. Because the eigen vectors show the principal directions of electromagnetic media, they can be used as standard spaces. Based on the spaces, we get the modal equations of static electromagnetic fields by converting the classical Maxwell's vector equation to the eigen Maxwell's scalar equation, each of which shows the existence of an static electromagnetic field. For example, there is only one kind of static electromagnetic field in isotropic crystal, which is identical with the classical result; there are two kinds of static electromagnetic fields in uniaxial crystal; three kinds of static electromagnetic fields in biaxial crystal and three kinds of distorted static electro- magnetic fields in monoclinic crystal. All of these new theoretical results need to be proved by experiments in the future.

After the text edit has been completed, the paper is ready for the template. Duplicate the template file by using the Save As command, and use the naming convention prescribed by your conference for the name of your paper. In this newly created file, highlight all of the contents and import your prepared text file. You are now ready to style your paper; use the scroll down window on the left of the MS Word Formatting toolbar.

\section{REFERENCES}

[1] J. Kong, "Theory of Electromagnetic Waves," WileyInterscience, New York, 1975.

[2] D. Frankl, "Electromagnetic Theory," Prentice-Hill, New Jersey, 1986.

[3] M. Zuniga and J. Kong, "Modified Radiative Transfer Theory for a Twolayer Random Medium," Journal of Applied Physics, Vol. 51, August 1987, pp. 5228-5244.

[4] V. Tamoikin, "The Average Field in a Medium Having Strong Anisotropic Inhomogeneities," Radiophysics and Quantum Electronics, Vol. 14, No. 2, March 1987, pp. 228-233.

[5] K. Chari and P. Silvester, "Finite Elements in Electric and Magnetic Field Problems," John Wiley and Sons, New York, 1980.

[6] S. Guo, "An Eigen Theory of Electromagnetic Waves Based on the Standard Spaces," International Journal of Engineering Science, Vol. 47, No. 3, May 2009, pp. 405412. 